\title{
Comparison of Proximal Injections of Local Anesthetics with Distal Injections in Intravenous Block
}

\author{
Ahmad Shokrzadeh \\ Assistant professor of Anesthesiology, Rajaie Hospital \\ Qazvin University of Medical Sciences, Qazvin, Iran
}

Tel: 98-281-333-5800 E-mail: ashokrzadeh@qums.ac.ir

Afsoun Seddighi (Corresponding author)

Assistant professor of Neurosurgery, Rajaie Hospital

Qazvin University of Medical Sciences, Qazvin, Iran

Tel: 98-281-333-5800Ｅ-mail: aseddighi@qums.ac.ir

\author{
Amir Saied Seddighi \\ Neurosurgeon, Assistant Professor of Neurosurgery, Shohada Tajrish Hospital \\ Beheshti University of Medical Sciences, Tehran, Iran \\ Member of Neurofunctional Research Center of Beheshti University \\ Tel: 98-218-826-5188Ｅ-mail: a_sedighi@sbmu.ac.ir
}

\begin{abstract}
Background: Perfect pain management is one of the most essential problems in anesthesia. Intravenous block is considered a simple anesthetic method to reduce extremity pain. This study is aimed to determine the effect of proximal injection compared with distal injections to avoid the problems related to be in the vicinity of the surgical field and making use of the larger proximal veins instead of the tiny distal vessels.

Methods: In this randomized double blind clinical trial, the patients with traumatic injuries of upper extremities referred to Rajaie Hospital from October 2003 to February 2004 were studied. The cases were divided blindly in to two groups. In one group injections were performed proximally and for the second group distally.
\end{abstract}

Results: This study was based on the data from 100 serial patients. The average time to achieve analgesia was $4.17 \pm 0.57$ minutes for the patients who received distal injections and for the cases that had proximal injection the mean time for analgesia was $5.19 \pm 0.55$. There was no difference in the rate of haematoma formation between the two groups.

Conclusions: In intravenous nerve block, the injection of local anesthetic agents in the proximal parts of the limbs is a safe method and is considered as effective as distal injections.

Keywords: Analgesia, Proximal, Distal, Intravenous, Block

\section{Introduction}

One of the most important issues in the field of Anesthesiology is to provide proper and effective analgesia with the lowest risk of side effects in each operative procedure (Barash 1996). Sharp injuries of upper extremities constitute a wide majority of operations performed due to traumatic injuries. These procedures can be performed not only under general anesthesia but also with regional analgesia which is obviously less hazardous for the patients especially for the elderly. So the use of local anesthetic methods is more popular (Blyth 1995).

Intravenous nerve block (Bier block) is a very simple method which can be used in every medical center with a high index to achieve analgesia in the desired area. The only requisiteness is to find an appropriate vein to perform the injection. This may be sometimes problematic due to limitations to find the proper vein with suitable diameter. It may also be troublesome to work close to the field of the vascular surgeon and struggle with the scrub nurse or the surgical team about the limitation of the local space to work. So this study is aimed to 
determine the effect of proximal injection compared with distal injections to avoid the problems related to being in the vicinity of the surgical field and making use of the larger proximal veins instead of the tiny distal vessels.

\section{Methods}

In this randomized clinical trial, the patients with traumatic injuries of upper extremities referred to Rajaie Hospital from October 2003 to February 2004 were studied. The exclusion criteria were as follows: Age under 25 and more than 45 years, loss of consciousness, drug abuse, Alcohol consumption, past history of peripheral neuropathy, use of analgesics 12 hours before entrance to the operating room. Informed consents were signed by all the selected patients. The patients were offered to choose between two colors, red and green. Those who selected green were classified as the first group and considered for proximal injection. Those who selected red were considered for distal injection. All of the patients received $0.04 \mathrm{mg}$ Midazolam and $1 \mathrm{mg} / \mathrm{kg} \mathrm{Fentanyl.}$ After one minute, $50 \mathrm{ml}$ Lidocaine $20 \%$ was injected by a 22 gauge needle. In the first group the anesthetic injected in the antecubital fossa veins and in the second group the injections performed from the dorsal veins of the hands. The depth of analgesia was recorded every 20 second for a period of 10 minutes or until the patient reported complete painlessness. The cases that did not achieved painlessness in 10 minutes or needed more analgesics were considered as the failed cases. The time of beginning of analgesia following injection was recorded for every case and compared. For statistical analysis $\chi^{2}$ was used.

\section{Results}

This study was based on experimental results of 100 patients with traumatic injuries of upper extremities referred to Rajaie Hospital from October 2003 to February 2004. In the first group who received distal injections 37 cases were male and 13 were female. In the second group who received proximal injections, 32 patients were male and 18 were female. The difference between sex distributions between the two groups was not statistically significant. One case $(2 \%)$ from the first group and 2 cases (4\%) from the second group showed failure of pain control. This difference was not significant statistically. Local haematoma occurred in one case $(2 \%)$ among the patients who received distal injections and in 2 patients (4\%) among the other group. There was no difference in the rate of haematoma formation between the two groups. The average time to achieve analgesia was $4.17 \pm 0.57$ minutes for the patients who received distal injections and for the cases that had proximal injection the mean time for analgesia was $5.19 \pm 0.55$.

\section{Discussion}

Bier block anesthesia is an intravenous regional anesthesia technique in which an extremity (generally an arm) is made numb for surgery by injecting a local anesthetic solution into a vein after the blood has been squeezed out of the extremity and a tourniquet has been placed on it. The tourniquet prevents the local from leaving the extremity and blood from entering it, giving the patient a numb (anesthetic) extremity and the surgeon a bloodless field to work in. The technique is named for August Bier (Miller 2000). The advantages of the technique is that it is simple to perform, and provides a bloodless field that is easy to work in and minimizes blood loss (Numm 1989). Regional analgesia is obviously less hazardous for the patients compared with general anesthesia. So the use of local anesthetic methods is more popular (Brown 1996). Our study showed that in intravenous nerve block, the injection of local anesthetic agents in the proximal parts of the limbs is a safe method and is considered as effective as distal injections (Rogger 1997). Conventional studies suggested the veins of the dorsal hand are better options for injection (Vincent 1993).

Blyth and colleagues suggested that proximal veins can be used effectively for nerve block in Callis fracture (Blyth 1995). Proximal veins are more convenient to use due to larger diameter and appropriate distance from the surgical field. It can produce the desired level of analgesia without a significant harm for the patients.

In our study the beginning of analgesia was a little bit later compared with distal injection but it was not so problematic.

\section{Conclusions}

In intravenous nerve block, the injection of local anesthetic agents in the proximal parts of the limbs is a safe method and is considered as effective as distal injections.

\section{References}

Barash PG, Cullen FB. (1996). Clinical anesthesia. $3^{\text {rd }}$ ed. New York: Lippincott. Raven, 27.645-683.

Blyth MJ, Kinninmonth AW, Asante DK. (1995). Bier Block: A change of injection site. J Trauma, 39 (4): 725-8.

Brown DL. (1996). Atlas of Anesthesia. $2^{\text {nd }}$ ed. Philadelphia: Saunders: 68-9. 
Miller RD. Anesthesia. (2000). $5^{\text {th }}$ ed. Philadelphia: Churchill Livingstone: 1541.

Numm UB. (1989). General anesthesia. $5^{\text {th }}$ ed. New York: Butter Wort, 1066-7.

Roger MC, Tiker JH. (1997). Principles and practice of anesthesia. $4^{\text {th }}$ ed. New York: Mosby, Vol 2, 1331.

Vincent JC. (1993). Principles of anesthesia. $3^{\text {rd }}$ ed. Philadelphia: Lea \& Febiger, Vol 1, 794-8. 